\title{
ESTUDOS “IN VITRO" DOS NÍVEIS DE RESISTENCIA DO PLASMODIUM FALCIPARUM A DROGAS, DE 1983 A 1986
}

M. A. V. SANTOS (1), A. A. CoUTo (1), S. G. OLIVEma (1) \& V. E. Rosario (2)

\section{R E S U M O}

Foram realizados 171 testes de sensibilidade (microtécnica) com cepas de Plasmodium falcipartm da Região Amazônica brasileira para cloroquina, mefloquina, amodiaquina e quinino. Os testes tiveram duração de 24 horas com as drogas preparadas na hora da realização de cada teste. Os resultados mostraram elevada resistência a cloroquina (83\%) e sensibilidade em quase a totalidade das amostras testadas para mefloquina $(97,7 \%)$. Para amodiaquina e quinino observou-se sensibilidade em $51,0 \%$ e $56,5 \%$ das cepas, respectivamente.

Este estudo demonstra a emergência de um possivel foco de resistência do Plasmodium falciparum a mefloquina, em Tucurui.

UNITERMOS: Malária; P. falciparum; Resistência; Mefloquina.

\section{N T R O D U G A $\mathbf{A}$}

Os problemas de saúde existentes na Amazônia, apresentam a malária como líder de uma lista de doenças infecciosas parasitárias que atinge $o$ homem da região em todo seu potencial produtivo. A epidemiologia desta doença é influenciada pelo grande número de pessoas que circulam na região, decorrentes da implantação de projetos agropecuários, agroindustriais e de mineração $1 ; 10,13,19$.

O grave proßlema da expansão da malária, associada ao aumento de casos de resistência dos plasmódios aos quimioterápicos utilizados nos esquemas clássicos de tratamento, tem sido frequientemente referido em vários trabaIhos $9,10,13,16,19,22$.

Diante deste quadro, vários grupos de pesquisadores, com o objetivo de uma avaliação da sensibilidade e resistência do Plasmodium falciparum, desenvolveram estudos "in vivo" e "in vitro", comprovando a existência de cepas de Plasmodium falciparum resistentes a alguns antimaláricos como amodiaquina, quinino, pirimetamina + sulfadoxina ou associação destes, e, principalmente, à cloroquina $1,3,4,6,18,23,24$.

A existência de cepas resistentes às drogas usuais, em muitas partes do mundo, vem estimulando a pesquisa para a obtenção de novas drogas antimaláricas mais eficazes contra as cepas resistentes 2,$8 ; 12,26$. Por outro lado, estudos são também desenvolvidos no sentido da utilização de combinações de drogas alternativas para a prevenção e tratamento da ma. lária 14,15 .

O Programa Malária dơ Instituto Evandro Chagas - FSESP, desenvolvendo estudos de caracterização do Plasmodium falciparum, realizou no período de 1983 a 1986, microtestes de sensibilidade a drogas em cepas coletadas em diversas localidades da Região Amazônica brasileira, com base na técnica descrita por

(1) Instituto Evandro Chagas (FSESP) - Programa Malária.

Av. Aimirante Earroso, 492. Caixa Postal 621. CEP 66050 Belém, PA., Brasil.

(2) Biomedical Research Institute, Rockville, Maryland, USA. 
SANTOS, M. A. V.; COUTO, A. A.; OLIVEIRA, S. G. \& ROSARIO, V. E. - Estudos "in vitro" dos níveis de resistan. cia do Plasmodium falciparum a drogas, de 1983 a 1986. Rev. Inst. Med. trop. São Paulo, 29:346-353, 1986.

RIECKOMANN et al. ${ }^{17}$, teve como objetivo inicial uma avaliação global da região.

O presente trabalho descreve o estiudo de 171 cepas de Plasmodium falciparum, incluin. do 65 testes, os quais foram publicados anteriormente ${ }^{27}$.

\section{MATERIAL E METODOS}

As amostras sangiuineas infectadas com Plasmodium falciparum (cepas), foram cole tadas de pacientes em diversas localidades da Regiäo Amazônica (Fig. 1) registrando-se a história clínica de cada um. Estas informa côes foram obtidas pela entrevista com os pacientes no momento da coleta, ou a partir dos dados do prontuário hospitalar.

As amostras (5 ml de sangue infectado) foram transportados a temperatura ambiente, em seringas heparinizadas, ou, se provenien. tes de localidades distantes do labaratório, em tubos de $15 \mathrm{ml}$ com igual volume de meio de cultivo RPMI 1640 (GIBCO), adicionado de bicarbonato de sódio e sôro humano tipo " $A$ " a $10 \%$. As amostras nessas condiçōes mantém os parasitas vivos até 72 horas. Nas localidades

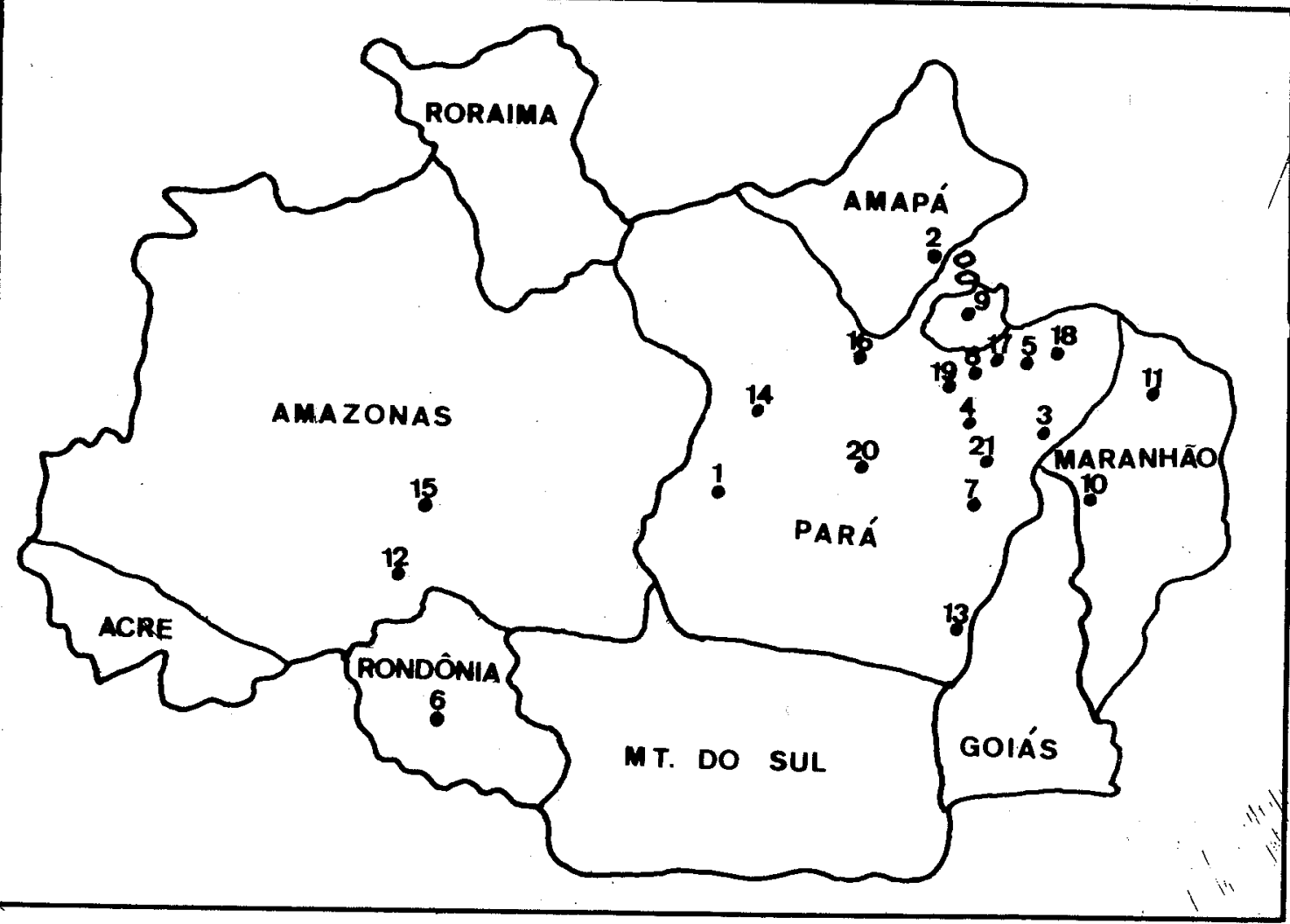

Fig. 1 - Localizacão das areas de coleta das cepas

$$
\begin{aligned}
& 01 \text { - ITATIUEA (PA) - } 36 \\
& 02 \text { - MACAPA (AP) - } 26 \\
& 03 \text { - PARAGOMINAS (PA) - } 22 \\
& 04 \text { - TUCURUI (PA) }-21 \\
& 05 \text { - ACARA (PA) - } 15 \\
& 06 \text { - ARIQUEMES (RO) - } 13 \\
& 07 \text { - MARABA (PA) - } 12 \\
& \text { 08 - MOJU (PA) - } 05 \\
& 09 \text { - MARAJO (PA) - } 02 \\
& 10 \text { - IMPERATRIZ (MA) - } 1 \\
& 11 \text { - PINHEIROS (MA) - } 1
\end{aligned}
$$

$$
\begin{aligned}
& 12 \text { - HUMAITA (AM) - } 02 \\
& 13 \text { - CONC. DO ARAGUAIA (PA) - } 02 \\
& 14 \text { - SANTAREM (PA) - } 01 \\
& 15 \text { - JUMAS (AM) } 01 \\
& 16 \text { - TAILANDIA (PA)-01 } \\
& 17 \text { - CAMETA (PA) - } 01 \\
& 18 \text { - TOME-AGU (PA) - } 01 \\
& 19 \text { - IGARAPE-MIRI (PA) - } 01 \\
& 20 \text { - ALTAMTRA (PA) - } 01 \\
& 21 \text { - RIO JACUNDA (PA) - 01 } \\
& \text { - ORIGEM DESCONHECIDA (PA) - } 05
\end{aligned}
$$


SANTOS, M. A. V.; COUTO, A. A.; OLIVEIRA, S. G. \& ROSARIO, V. E. - Estudos "in vitro" dos niveis de résisténcia do Plasmodium falciparum a drogas, de 1983 a 1986. Rev. Inst. Med. trop. São Paulo, 29:346-353, 1986.

de dificil acesso e distantes do laboratório em Belém (PA), as mostras após colhidas, foram criopreservadas em Dimetilsufóxido (DMSO) a $20 \%$, para posterior processamento (descongelamento e cultivo contínuo) para a realização dos microtestes.

Para a realização do microteste, a parasitemia adequada é de 0,1 a $1,0 \%$. Em presença de parasitemias elevadas, procedeu-se a diluição utilizando-se hemácias lavađas (tipo "0"), enquanto que para parasitemias mais baixas, realizou-se o cultivo contínuo "in vitro", de acordo com a técnica de TRAGER \& JENSEN, até obtenção đa parasitemia ideal ${ }^{25}$.

Das 171 cepas testadas, 145 foram mantidas em cultivo por períodos que variaram entre 3-15 dias, e as 26 restantes foram testadas logo após a coleta e chegada do sangue dos pa cientes ao laboratório, sendo a origem da infecção e história clínica por vezes descritas de acordo com a informação verbal do paciente. O cultivo contínuo não apresentou marcante multiplicidade morfológica das formas eritrocitárias, ou seja, a presença simultânea de anéis, trofozoitos e esquizontes, pelo que os microtestes foram realizados com as amostras contendo predominância de anéis.

Todas as amostras de sangue foram lavadas em meio RPMI-1640 (GIBCO), adicionado de bicarbonato de sódio a $10 \%$, Após 2 lavagens (3.000 r.p.m./5 min), o sangue foi diluído em igual volume de meio de cultivo completo: $88 \mathrm{ml}$ de RPMI-1640 (GIBCO) $+2 \mathrm{ml}$ de bicarbonato de sódio e soro humano "A" a $10 \%$, utilizou-se microplacas de titulação de polietileno (Petécil), de fundo raso, colocando-se 0,01 ml da suspensão de sangue nos orifícios da placa, aos quais adicionou-se $0,1 \mathrm{ml}$ do meio completo RPMI com diversas concentraçōes da droga, tal como se segue:

1) $0-1,0-2,0-4,0-6,0-8,0-16,0$ - 32x10-8 M (Difosfato de Cloroquina)

2) $0-0,5-1,0-2,0-4,0-6,0-8,0$ - 16,0×10-8 M (Mefloquina)

3) 0 - $-0,25-0,5-1,0-2,0-4,0-8,0$ - 16,0x10-8 M (Amodiaquina)

4) $0-3,9-7,8-15,7-31,25-62,5-125$ - 250x10-8M (Sulfato de Quinino)
As amostras foram preparadas a partir de soluções "stock" (OMS-Geneve) e as dosagens foram preparadas no momento em que se iniciou o teste. Em trabalho anterior ${ }^{2 \pi}$, foram utilizadas cepas controle testadas pelo mesmo método no Instituto Animal Genetics, Edinburgh, Grã-Bretanha. Para os restantes testes foram usadas cepas controles padronizadas pe. lo Programa Malária do Instituto Evandro Chagas, como IEC-50/83 e IEC-132/83.

Nem todas as amostras foram submetidas a testes com todas as drogas porque, inicialmente, este trabalho consistiu na analise de testes realizados apenas com cloroquina e mefloquina.

\section{RESULTADOS}

Na tabela I apresentamos o número tctal de amostras testadas por localidade e ano da coleta. Nas tabelas II e III os resultados coos microtestes estão distribuidos na forma de MIC (Concentração Mínima Inibidora) por ano e nas diferentes dosagens testadas para cada droga:

T A B $\mathbf{E} \mathbf{L}$ A I

Número de microtestes, realizzados por ano e por localidade de origem das cepas

\begin{tabular}{|c|c|c|c|c|c|}
\hline Localidade & 1983 & 1984 & 1985 & 1986 & Total \\
\hline Itaituba (PA) & 9 & 16 & 6 & 5 & 36 \\
\hline Macapa (AP) & 4 & 7 & 6 & 9 & 26 \\
\hline Paragominas (PA) & 15 & $\mathbf{2}$ & $\mathbf{5}$ & - & 22 \\
\hline Tucuruí (PA) & 8 & 6 & 7 & - & 21 \\
\hline Acará (PA) & 一 & 11 & $\mathbf{3}$ & 1 & $\mathbf{1 5}$ \\
\hline Ariquemes (RO) & 一 & $\mathbf{5}$ & 8 & - & 13 \\
\hline Maraba (PA) & $\mathbf{2}$ & 4 & 2 & 4 & 12 \\
\hline Mojú (PA) & 2 & 2 & 1 & - & 5 \\
\hline Marajo (PA) & 1 & - & 1 & 一 & 2 \\
\hline Imperatriz (MA) & - & 一 & $\mathbf{1}$ & 一 & 1 \\
\hline Pinheiros (MA) & 1 & - & - & - & 1 \\
\hline Humaitá (AM) & - & - & $\mathbf{2}$ & - & $\mathbf{2}$ \\
\hline Conc. do Araguaia (PA) & - & - & - & $\mathbf{2}$ & $\mathbf{2}$ \\
\hline Santarém (PA) & $\mathbf{1}$ & $\longrightarrow$ & - & 一 & 1 \\
\hline Jumas (AM) & 1 & - & - & 一 & 1 \\
\hline Tailândia (PA) & 1 & - & - & - & 1 \\
\hline Cametá (PA) & - & 1 & 一 & 一 & 1 \\
\hline ToméAcí (PA) & - & 1 & 一 & - & 1 \\
\hline Igarape-Miri (PA) & - & 1 & - & $\longrightarrow$ & 1 \\
\hline Altamira (PA) & - & $\longrightarrow$ & 1 & 一 & 1 \\
\hline Rio Jacunda (PA) & - & - & 1 & 一 & 1 \\
\hline * & 4 & 1 & 一 & 一 & 5 \\
\hline Total & 49 & 57 & 44 & 21 & 171 \\
\hline
\end{tabular}

- Cepas cujos pacientes não souberam informar o loca de infecção, mas afirmam que nāo sairam do Estado do Para. 
SANTOS, M. A. V.; COUTO, A. A.; OLIVEIRA, S. G. \& ROSário, V. E. - Estudos "in vitro" dos niveis de resistên cia do Plasmodium faloiparum a drogas, de 1983 a 1986. Rev. Inst. Med. trop. São Paulo, 29:346-353, 1986.

T A B E I A II

Caracterização de cepas de $\mathbf{P}$. falciparum através de testes de sensibilidade

Distribuição dos resultados de março/83 a junho/86

\begin{tabular}{|c|c|c|c|c|c|c|}
\hline Drogas & MIC* & 1983 & 1984 & 1985 & 1986 & $\begin{array}{l}\text { Total de amos } \\
\text { tras inibidas }\end{array}$ \\
\hline Cloroquina & 1 & 0 & 0 & 0 & 0 & $\mathbf{0}$ \\
\hline \multirow[t]{6}{*}{$\times 10.8 \mathrm{M}$} & 2 & 0 & 0 & 0 & 0 & 0 \\
\hline & 4 & 1 & 0 & 1 & 0 & 2 \\
\hline & 6 & 11 & 8 & 5 & 3 & 27 \\
\hline & 8 & 6 & 12 & 8 & 3 & 29 \\
\hline & 16 & 12 & 16 & 15 & 10 . & 53 \\
\hline & 32 & 19 & 21 & 15 & 5 & 60 \\
\hline Total de Cepas & & 49 & 57 & 44 & 21 & 171 \\
\hline Mefloquins & 0,5 & 30 & 6 & 9 & 2. & 47 \\
\hline \multirow[t]{6}{*}{$\times 10.8 \mathrm{M}$} & 1 & 11 & 26 & 22 & 6 & 65 \\
\hline & 2 & 8 & 14 & 4 & 10 & 36 \\
\hline & 4 & 0 & 7 & 4 & 3 & 14 \\
\hline & 6 & 0 & 1 & 2 & o & 3 \\
\hline & 8 & 0 & 1 & 3 & O & 4 \\
\hline & 16 & 0 & 0 & 0 & 0 & $\mathbf{0}$ \\
\hline Total de Cepas & & 49 & $55^{* *}$ & 44 & 21 & 169 \\
\hline Amodiaquina & 0,25 & 1 & 0 & 3 & 0 & 4 \\
\hline \multirow[t]{6}{*}{$\mathrm{x} 10.8 \mathrm{M}$} & 0,5 & 0 & 4 & 5 & 2 & 11 \\
\hline & 1 & 3 & $\mathbf{8}$ & 12 & 4 & 27 \\
\hline & 2 & 1 & 14 & 8 & 4 & 27 \\
\hline & 4 & 4 & 20 & 6 & 7 & 37 \\
\hline & 8 & 7 & 6 & 8 & 3 & 24 \\
\hline & 16 & 2 & 5 & 2 & 1 & 10 \\
\hline
\end{tabular}

\begin{tabular}{lcccccc}
\hline Total de Cepas & & 18 & 57 & 44 & 21 & 140 \\
\hline Quinino & 3,9 & 0 & 0 & 0 & 0 & 0
\end{tabular}

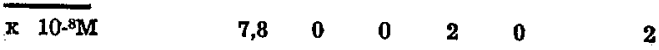

$\begin{array}{rrrrrr}15,6 & 1 & 11 & 9 & 2 & 23\end{array}$

$\begin{array}{lllllll}31,25 & 9 & 24 & 16 & 5 & 54\end{array}$

$\begin{array}{llllll}62,5 & 6 & 17 & 10 & 9 & 42\end{array}$

$\begin{array}{rrrrrr}125 & 2 & 1 & 6 & 5 & 14\end{array}$

$\begin{array}{llllll}250 & 0 & 4 & 1 & 0 & 5\end{array}$

Total de Cepas

$\begin{array}{llll}18 & 57 & 44 & 21\end{array}$

140

* Concentração Mínima Inibidora da droga, capaz de inibir o crescimento do parasito.

* Dois testes de Mefloquina foram perdidos por deficiência da coloração das lâminas.

a) Cloroquina: Das 171 amostras testadas 142 foram inibidas acima de $8 \times 10^{-8} \mathrm{M}$, sendo que apenas 2 foram inibidas a $4 \times 10^{-8} \mathrm{M}$ ( 1 de $\mathrm{Ma}$ capá e 1 de Ariquemes-Ro) e 27 foram inibidas a $6 \times 10^{-8} \mathrm{M}$ (7 de Paragominas-PA, 4 de Itaituba-PA, 3 de Tucuruí-PA, 3 de Ariquemes-RO, 2 de Acará-PA, 2 de Macapá-AP, 1 de Mojú-PA, 1 de Santarém-PA, 1 de Tailânđia-PA, 1 de $\mathrm{Ma}$ raba-PA, 1 de Imperatriz-MA e 1 de PinheirosMA.).
Portanto, para cloroquina, detectou-se $83 \%$ de resistência "in vitro".

b) Mefloquina: Das 169 amostras testadas, e no mesmo periodo de 1983-1986, 142 foram inibidas a doses até $4 \times 10^{-8} \mathrm{M}$. Acima desta dosagem 3 amostras (Tucurui, Marajó e Acará-PA) foram inibidas a $6 \times 10^{-8} \mathrm{M}$ e 4 amostras (todas de Tucuruí-PA) foram inibidas a $8 \times 10^{-8} \mathrm{M}$, num total de 7 exibindo resistência "in vitro" a esta droga.

c) Amodiaquina e Quinino: Grande variação na resposta aos testes foi observada para am, bas as drogas. Das 10 amostras inibidas. a mais elevada dose $\left(16 \times 10^{-8} \mathrm{M}\right)$ de amodiaquina, 4 eram provenientes de Itaituba, 4 de Tucuruí-PA e 2 de Macapá-AP. Para quinino 5 amostras (3 de Tucuruí, 1 de Itaituba-PA e 1 de Ariquemes-RO) foram inibidas apenas na dose mais elevada $\left(250 \times 10^{-8} \mathrm{M}\right)$, mas também de Itaituba e Tucurui-PA encontram-se amostras sensiveis à doses baixas $\left(7,8 \times 10^{-8} \mathrm{M}\right)$.

\section{DISGUSSAO E CONGLUSOES}

Estes estudos efetuados no periodo de 1983-1986 cobriram 20 localidades, sendo a maioria no Estado do Pará e as restantes em Macapá (Território Federal do Amapá), Humaitá (Estado do Amazonas) e Ariquemes (Estado de Rondônia). O número de amostras coletadas foi variado e irregular. Por exem. plo: de apenas 3 localidades, Itaituba, Macapá - Marabá foi coletado material continuamente, num total, respectivamente, de 36,26 e 12 amostras.

Em grande número de localidades apenas um reduzido número de amostras foi analisado, mas nenhuma apresentou elevada resistência a qualquer das drogas testadas. Apesar do número de amostras ter sido reduzido, seria sempre de interesse a observação de- uma única amostra, com elevada resistência, que pudesse indicar ser aquela localidade possivel foco de parasitas não susceptíveis a dada droga.

Sobre todas as cepas testadas, observamos que sempre que uma cepa apresentou resistência ao quinino ou apresentou resistência a amodiaquina, esta mesma cepa apresentou também resistência a cloroquina. Porém, nem 


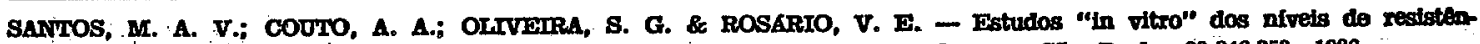
cla do Pastinodium falciparum a drogas, de 1983 a 1986. Rev. Inst. Med. trop. Säo Paulo, 29:346-353, 1986.

T. A $\mathbf{B}$ E I A III

Distribuilęăo gráfica de 171 microtestes realizados nos anos do 1983, 1984, 1985 e 1986, para quatro drogas

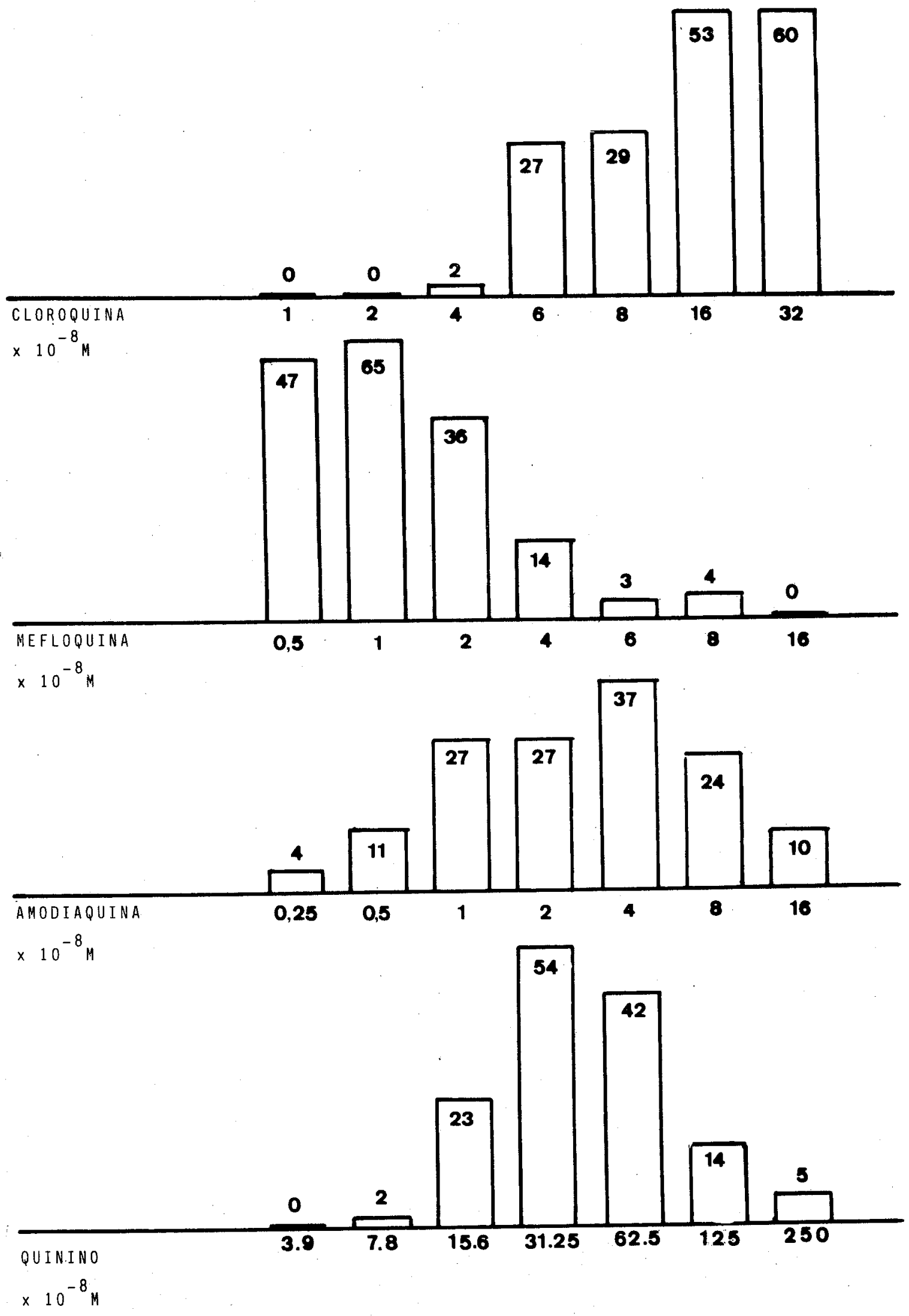


SANTOS, M. A. V.; COUTO, A. A.; OLIVEIRA, S. G. \& ROSARTO, V. E. - Estudos "in vitro" dos niveis de resisten cia do Plasmodium falciparum a drogas, de 1983 a 1986. Rev. Inst. Med. trop. São Paulo, 29:346-353, 1986.

sempre para ambos os casos o inverso foi verdadeiro.

Em Itaituba e Tucuruí-PA, encontrou-se o maior número de amostras mais resistentes às drogas testadas, isto é, apenas inibidas por doses mais elevadas utilizadas nos testes. A existência de focos de resistência à mefloquina em Tucurui-PA, numa região de elevado movimento migratório, e onde existe apenas um vetor de transmissão (ARRUDA et al 1986), é causa de preocupação, sobretudo porque esta droga não é ainda de uso generalizado no país; neste caso atuaria como agente seletivo de disseminação de resistência, com dramáticas consequiências.

Em relação a mefloquina, outros autores indicaram respostas distintas "in vivo", como SOUZA (1983), cujos pacientes responderam com sucesso ao tratamento, BOULOS et al. (1985) e DI SANTI et al. (1986) que mencionam fracasso no uso desta droga. $O$ intervalo de tempo na obtenção de uns e outros resulta. dos, bem como a distinta origem dos pacientes pode justificar esta variação. Grande parte dos pacientes envolvidos no Projeto Mefloquina (SOUZA, 1983), eram provenientes de Paragominas-PA, enquanto que os trabalhos realizados por BOULOS et al. e DI SANTI et al., eram de Rondônia e Amazonas, respectivamente $7,8,21$.

Em testes "in vitro" com a mefloquina e outras drogas, elevados níveis de resistência têm sido observados (ALECRIM et al., 1986) $12,2,3,4$.

Os microtestes devem ser utilizados para estudos longitudinais que permitam, para uma maior amostragem, detectar se nos períodos de menor transmisão existe, por exemplo, relação com presença de parasitas de maior ou menor resistência a alguma droga, ou se, no caso de Capanema, onde Plasmodium falciparum pode ser transmitido por. A. osvaldoi, (ARRUDA et al., 1986), existe um padrão de resistência e mesmo caracterização dos parasitas, sendo estes, diferentes do resto da Regiăo Amazônica. A longo prazo, e caso a cloroquina deixe de ser utilizada na região, estes microtestes permitirão avaliar uma diminuição do nivel de resistência a esta droga como foi descrito para o Sudoeste Asiático 11,20.
O elevado movimento migratorio identifi. cado nas áreas de desenvolvimento do país, sobretudo na região acima citada, apenas fa vorece a expansão da resistência às drogas na presença do vetor.

Torna-se importante estudos mais regulares e periodicos em um número mais reduzido de localidades distintas para se compreender melhor o problema de instalação e expansão de resistência na região amazônica.

\section{SUMMYARY}

"In vitro" studies on the levels of Plasmodium falciparum drug resistance from 1983 to 1986.

Drug resistance tests 'were performed with 171 strains of $P$. falciparum obtained from the Amazon Region, using chloroquine, mefloquine, amodiaquine and quinine. Tests lasted about 24 hours and the drug solutions were prepared just before use. Results show high percentage of resistance to chloroquine (83\%) Whereas nearly all strains were shown to be sensitive to mefloquine $(97.7 \%)$. For both amodiaquine and quinine sensitivities were of $51.0 \%$ and $56.5 \%$ respectively,

The present study demonstrates that re sistance to mefloquine may be starting in Tucuruí, Pará.

\section{AGRADECIMENTOS}

Este trabalho contou com o apoio do CNPq, FINEP, SUCAM e OMS-TDR. Agradecemos também ao Dr. José Maria de Souza Projeto Mefloquina - Hospital Barros Barre to - Belém (PA), pelo envio de cepas de aigumas localidades do Estado do Pará.

\section{REFERENGIAS BIBLIOGRAFICAS}

1. ALECRIM, M. G. - Estudo da resistência do P. falciparum às drogas antimaláricas "in vitro" e "in vivo" na Amazônia. Brasilia, 1981. (Dissertação de mestrado - Universidade de Brasilia).

2. ALECRIM, M. G.; ALECRIM, W. D.; ALBUQUERQUE, B. C. \& SHVA, E. E. - Fracasso no tratamento da maĺria (P. falciparum) com novas árogas antimalaricas. In: CONGRESSO DA SOCIFDADE BRASILEIRA DE MEDICINA TROPICAL, 22., Belo Horizonte, 1986. Programa e resumos. Belo Horizonte, Associaçāo Médica de Minas Gerais, 1986. p. 91. 
SANTOS, M. A. V.; COUTO, A. A.; OLIVEIRA, S. G. \& ROSARIO, V. E. - Fstudos "in vitro" dos niveis de resisteancia do Plasmodium taleiparum a drogas, de 1983 a 1986. Rev. Inst. Med. trop. São Paulo, 29:346-353, 1986.

3. AIECRIM, W. D.; ROBLES, C. R. Q.; REYS, S. \& NASCIMENTO, A. P. - Testes "in vivo" de resistência do P. falciparum à cloroquina, amodiaquina e sulfadoxina + pirimetamina na Amazónia. In: CONGRESSO DA SOCIFDADE BRASILEIRA DE MEDICINA TROPICAL, 22., Belo Horizonte, 1986. Programa e resumos. Belo Horizonte, Associação Médica de M1nas Gerais, 1986. p. 90.

4. ALECRIM, W. D.; ALECRIM, M. G.; ALEUQUERQUE, B. C. \& SAMPAIO, G. - Testes "in vivo" da resistência do P. falciparum ao quinino, sulfadoxina + pirimetamina $\theta$ sufadoxina + pirimetamina + amodiaquna. In: CONGRESSO DA SOCIEDADE BRASIIEIRA DE MEDICINA TROPICAL, 22., Belo Horizonte, 1986. Programa e resumos: Belo Horizonte, Associação Médica de Minas Gerais, 1986. p. 89.

5. ARRUdA, M. E.; CaRValHo, M.; NUSSENZWEIG, R.; MARACIC, M.; FERREIRA, A. W. \& COCHRANE, A. H. - Potencial vectors of malaria and their different susceptibility to Plasmodium falciparum and Plasmodium vivax in Northern Brazil identified by im. munoassay. Amer. J. trop. Med. Hyg., 35: 873-881, 1986.

6. BOULOS, M.; DI SANTI, S. M.; NEVES, V. L. F. C.; Barata, L. C. B. \& DUTRA, A. P. - Correlaৎ̧ão "in vivo" - "in vitro" da resposta do P. tal. clparum ao quinino. In: CONGRESSO DA SOCIFDADE BRASILIFIRA DE MEDICINA TROPICAL, 22., Beto Horizonte, 1986. Programa e resumos. Belo Horizonte, Alssociação Médica de Minas Gerais, 1986. p. 89.

T. BOULOS, M.; SEGURADO, A. A. C.; DUTRA, A. P.; SHIROMA, M. \& FERREIRA, I. - Resistência do P. falciparum à associação mefloquina + sulfadoxina + pirimetamina. In: CONGRESSO DA SOCJFDADE IRASIIEIRA DE MEDICINA TROPICAL, 22., Belo Hor1zonte, 1986. Programa resumos. Belo Horizonte, Associação Médica do Minas Gerais, 1986. p. 88.

B. DI SANTI, S. M.; BOULOS, M.; NEVES, V. I. F. C.; DUTRA, A. P. \& BARATA, L. C. B. - Aparecimento e proliferação de resistência do $P$. falciparum à mefloquina. In: CONGRESSO DA SOCIFDADE BRASIINEIRA DE MEDICINA TROPICAL, 22., Belo Horizon. te, 1986. Programa e resumos. Belo Horizonte, Assoclação Médica de Minas Gerais, 1986. p. 89.

9. DI SANTI, S. M.; NEVES, V. L. F. C.; BOULOS, M.; DUTRA, A. P. \& BARATA, L. C. B. - Avaliaçäo da resistêncla do P. talciparum à cloroquina na amazônia brasileira. In: CONGRESSO DA SOCIFDADE BRASII.FIRA DE MEDICINA TROPICAL, 22., Eelo Horizonte, 1986. Programa e resumos. Belo forizonte, Associaçäo Médica de Minas Geraís, 1986. p. 88.

10. FigrRaroni, J. J.; SPEER, C. A.; HAYES, J. \& SU. ZUKI, M. - Prevalence of chloroquine resistant falciparum malaria in Brazillan Amazon. Amer. J. trop. Med. Hyg., 30: 526-530, 1980.

11. JACQUIFR, P.; DRUILAE, P.; FHLXX, H.; DIQUET, B. D. TIBO, L. - Is Plasmodium falciparum resis- tance to chloroquine reversible in absence of drug pressure? Lancet, 2: 270-271, 1985.

12. LOPEZ-ANTUNANO, F. J. \& WERNSDORFER, W. H. - In vitro response of chloroquine resistant Plasmo. dium falciparum to mefloquine. Bull. wid. Hith. Org., 57: $663-665,1979 .$.

13. MARQUES, A. C. - Situação atual da malária no Brasil. In: SEMINARIO AMAZONICO DE MEDICINA TROPICAL E SAÚDE PÚBLICA, 13., Belém (PA), 1986. Programa e resumos. (In press).

14. REYES, S. - Infecçōes maláricas por Plasmodium falciparum resistentes ao tratamento com cloroquina. Situação no. Erasil (1960-1981). Rev. bras. Malar., 33: 109-130, 1981.

15. PETERS, W. - Prevention of arug resistance in ro. dent malaria by the use of drug mixtures. Bull. Wid. Hth. Org., 51: 379-383, 1974.

16. PETERS, W. - Drug combinations. In: PETSRS, $W$. \& RICHARDS, W. H. G., ed. - Antimalarial drug $\mathbf{I}$. Current antimalarials and new drug developments. Berlin, Springer-Verlag, 1984. p. 225-236.

17. RIECKMANN, K. H.; SAX, L. J.; CAMPBELL, G. H. \& UREMA, J. F. - Drug sensitivity of Plasmodinm alciparum. An "in vitro" microtechnique. Lancet, 1: 22-23, 1978 .

18. RODRIGUES, D. P. - Casos de malária por Plasmodium falciparum resistentes ao tratamento pela cloroquina. Arq. Hig. Saúde puibl., 2b: 231-235, 1961.

19. ROSARIO, V. E. - Caracterização de cepas de Plasmodium falciparum do Brasil. Rev. Fundaçäo SESP, 28: 115-136, 1983.

20. SILVA, J. R.; LOPES, P. F. A.; FERREIRA, L. F. F.; MORTEO, R. \& NAVETRA, J. B. - Resistencia do Plasmodium talciparam a agá da cloroquina. Hos. pital, (Rio de J.), 60: 581-594, 1961.

21. SOUZA, J. M. - A phase II clinical trial of mefloquine in Braxilian male subjects. Bull. Wh. Hith, Org., 61: 815-829, 1983.

22. THAITHONG, S: - In: International Congress of Infection and Parasitology Diseases, 9., Munick, 1986. Abstract 957.

23. THAITHONG, S. - Clones of different sensitivities in drug-resistant isolates of Plasmodium falchparum. Bull. Wld. Fith. Org., 61: 709-716, 1984.

24. THATTHONG, S.; BEALF, G. H. \& CHUMTMONGKONKUL, M. - Suspectibility of Plasmodium falclparum to five drugs: a "in vitro" study of isolates mainty from Thailand. Trans, roy. Soc. trop. Med. Hys.. 77: 228-231, 198?. 
SANTIOS, M. A. V.; COUTTO, A. A.; OLIVEIRA, S. G. \& RaSARIO, V. E. - Estudos "in vitro" dos niveis de resisten. cia do Plasmodium falciparum a drogas, de 1983 a 1986. Rev. Inst. Met. trop. Sáo Paulo, 29:346-353, 1986.

25. TRAGER, W. \& JENSEN, J. B. - Human malaria parasites in continuous culture. Sclence, 193: 673-675. 1976.

26. WERNSDORFER, W. H. \& KOUZNESOV, R. L. Drug resistant malaris occurrence control and sur. veillance. Bull. Wh. Wlth. Org., 58: 341-352, 1980.
27. VASCONCELOS, M. A. \& ROSARIO, V. E. - Teste. de sensibilidade "in vitro" de amastras de Plasmo. dium falciparum da bacia amazonica (Erasil). Bev. bras. Malar., 35: 21-28, 1983.

Recebido para publicagion ene 2/6/1987. 